\title{
Risks and results of surgery
}

In deciding which United Kingdom hospitals in the new internal market of the National Health Service should get the contracts, and hence which institutions should be able to survive, the obvious solution (which has been suggested) is to look at the rate of survival of their patients. What could be simpler than publicising mortality figures and let market forces do the rest? Such league tables have been mooted. They started 8 years ago in the United States when the results of open heart surgery in hospitals providing Medicare were made public. ${ }^{1}$

The immediately obvious problem is that league tables assume that all hospitals are taking on cases of similar risk. They then reward low risk, however it is achieved. We know that the lowest risk is obtained in patients for whom the indications for operation are least pressing. Patients with stable angina, that is manifest only at higher work levels, with good ventricles, and fewer stenoses, have very low risk at surgery-but do they have anything to gain in prognosis or by a net improvement in symptoms? When managed conservatively they are relatively well, and relatively safe. Operation carries a low risk but also a small margin of benefit. Patients with the slightly higher risk at operation who are at highest risk if left without an intervention, have the most to gain. Valve surgery is a little different. There is a point, when the disease has run its course for too long, at which there are only marginal benefits to be gained from desperate end stage surgery. None the less, if only low risk patients with valve disease are taken on we are denying surgery to those with the most to gain.

A system that in isolation rewards low risk would, in the case of cardiac disease, go a long way towards ensuring that the wrong patients get operated upon. This was evident in American practice where the best of the local private hospitals doing low volumes of good risk coronary surgery could produce mortality figures close to zero in the boom years of the mid eighties while the nearby university hospitals were seeing rising mortality figures. I heard family practitioners using simple mortality figures as evidence that their "local guy" (who in this case had supplied the figures in the most direct, oral, and unascertainable form) was better than the university. He would say that, wouldn't he? It may have been true, but it ignored any difference in case mix.

The first serious attempt at risk stratification to reach a wide audience was that of Parsonnet, ${ }^{1}$ now so widely cited that "Parsonnet scoring" has almost become a generic term. The system consists of a simple additive model in which several simple and readily available descriptors or measurements can be totted up to produce an estimate or risk (table). These were derived from retrospective univariate analysis of cases over a five year period (1982 to 1987) and tested prospectively at four other hospitals. From the model, patients could be stratified into good risk $(0-4 \%)$, fair risk (5-9\%), poor risk $(10-14 \%)$, high risk (15-19\%), and extremely high risk ( $20 \%$ or more).

\begin{tabular}{|lc|}
\hline Parsonnet scoring & \\
Risk factor & Assigned weight \\
\hline Female & 1 \\
Obesity $>1 \cdot 5$ times ideal weight & 3 \\
Diabetes & 3 \\
Hypertension >140 mm Hg & 3 \\
Ejection fraction (\%): & \\
$>50$ & 0 \\
$30-49$ & 2 \\
$<30$ & 4 \\
Age (yr): & 7 \\
$70-74$ & 12 \\
$75-80$ & 20 \\
$\geqslant 80$ & 5 \\
First reoperation & 10 \\
Second reoperation & 2 \\
Preoperative IABP & 5 \\
LV Aneurysm (resected) & 10 \\
Emergency from PTCA or cath lab & 10 \\
Dialysis dependent & $10-50$ \\
Catastrophic states & $2-10$ \\
Rare circumstances & \\
Valve surgery: & 5 \\
Mitral & 8 \\
PA $\geqslant 60$ mm Hg & 5 \\
Aortic & 7 \\
Gradient >120 mm Hg & 2 \\
CABG with valve & \\
\hline
\end{tabular}

IABP, Intra-aortic balloon pump; CABG, coronary artery bypass grafting.

This method was tested in British practice by Nashef and colleagues in Manchester. ${ }^{2}$ They confirmed that the system discriminated very well between these groups. The actual mortality figures in each stratum were consistently less than predicted by Parsonnet scores but the $95 \%$ confidence intervals of the proportions always included the predicted risk. In our own work we have found the same. The elements of the risk stratification are entered into our database before or at the time of operation. We have used Parsonnet data to compare the case mix of different surgeons within the unit ${ }^{3}$ and to monitor the risk of cases delegated to trainees. ${ }^{4}$ In all instances we have found, as did Nashef et al, that the actual mortalities in the groups ascend in step with the risk strata, but that we consistently achieve lower than predicted mortality in each group. Our conclusion is that this does not invalidate Parsonnet: it reflects the fact that we are comparing our present work (in a unit that has every intention of being in the $50 \%$ with "above average results") with figures that were achieved a decade ago.

Nashef et al wrote "Some surgeons and cardiologists are dismayed that the system excludes their 'favourite' risk factors: unstable angina, lung disease, and severity of coronary lesions are conspicuously absent but are difficult to define (unstable angina), not always documented (lung disease), or both (severity of coronary lesions). It is 
because such factors are excluded that the Parsonnet system is objective and easy to implement." I agree with that appraisal. There are always some who point to the case in which the prediction failed and the case that beat the odds or they claim that their clinical judgement is better than statistics. Let them hold those views. For our working purposes, Parsonnet is simple to use, robust, and useful for making informed comparisons between groups, and can always be overridden in the management of individuals.

More substantial was Spiegelhalter's criticism. ${ }^{5}$ Pointing to the consistent overestimate of risk, he faulted the use of univariate analysis and an additive model. As it stands, the predicted risks are too high and simply doing as well as them might not be doing as well as we should in the mid 1990s. The challenge is to refine the model and recalibrate it. However, Parsonnet scoring is not intended to be a crystal ball, gazed into to predict the outcome for an individual. The individual will be alive or dead, a hard fact disguised by some operational phrase such as "dichomotous actual outcome state". The purpose of scoring systems in this instance is to provide a means of redressing apparent differences in results by correcting for differential case mix.

The Regional Cardiothoracic Unit,

TOM TREASURE

St George's Hospital,

Blackshaw Road,

London SW17 OQT

1 Parsonnet V, Dean D, Bernstein AD. A method of uniform stratification of risk for evaluating the results of surgery in acquired adult heart disease. Circulation 1989;79(suppl I):3-12.

2 Nashef SAM, Carey F, Silcock MM, Oommen PK, Levy RD, Jones MT. Risk stratification for open heart surgery: trial of the Parsonnet system in a British hospital. BrMed $f$ 1992;305:1066-7.

3 Anderson J, Valencia O, Jackson R, Murday A, Treasure T, Smith EEJ, Parker DJ. Risk stratification: the case mix of individual surgeons within a cardiac surgical unit using the Parsonnet system [abstr]. Eur Heart $\mathcal{f}$ $1994 ; 15: 271$

4 Anderson J, Hunter S, Valencia O, Smith EEJ, Parker DJ. The reality of training in cardiac surgery. Br Heart $\mathcal{F}$ 1994;71 (suppl):P62.

5 Spiegelhalter DJ. Risk stratification of open heart surgery. BMF 1992; 305:1500. 